\title{
Inhaled Corticosteroid Use Among COPD Patients in Primary Care in Spain
}

\author{
Marc Miravitlles $\mathbb{D}^{1}$, Miguel Roman-Rodríguez $\mathbb{D}^{2,3}$, Xavier Ribera ${ }^{4}$, John Ritz ${ }^{5}$, José Luis Izquierdo $\mathbb{D}^{6,7}$ \\ On behalf of OPTI investigator's group
}

\begin{abstract}
'Pneumology Department, Hospital Universitari Vall d'Hebron/Vall d'Hebron Institut de Recerca (VHIR), Vall d'Hebron Barcelona Hospital Campus, CIBER de Enfermedades Respiratorias (CIBERES), Barcelona, Spain; ${ }^{2}$ Centro de Salud Dra. Teresa Pique, Mallorca, Spain; ${ }^{3}$ Instituto de Investigación Sanitaria de las Islas Baleares (IdISBa), Mallorca, Spain; ${ }^{4}$ Boehringer Ingelheim España, Sant Cugat del Vallés, Barcelona, Spain; ${ }^{5}$ Syneos Health Boehringer Ingelheim Pharmaceuticals Inc., Ridgefield, CT, USA; ${ }^{6}$ Department of Medicine and Medical Specialties, Universidad de Alcalá, Madrid, Spain; ${ }^{7}$ Pneumology Department, Hospital Universitario de Guadalajara, Guadalajara, Spain
\end{abstract}

Correspondence: Marc Miravitlles, Pneumology Department, Hospital Universitari Vall d'Hebron, P. Vall d'Hebron II9-129, Barcelona, ES-08035, Spain, Email marcm@separ.es

Purpose: Inhaled corticosteroids (ICS) are frequently used to treat chronic obstructive pulmonary disease (COPD) outside the current recommendations. Our aim was to describe ICS use in COPD patients and to identify factors associated with ICS use among COPD patients treated within primary care in Spain.

Patients and Methods: This was a cross-sectional, non-interventional and multicenter study of patients with COPD treated in primary care. Patient characteristics and exacerbations were described in terms of ICS use among the overall cohort, and among those with spirometry confirmed COPD (post-bronchodilator forced expiratory volume in 1 second $\left[\mathrm{FEV}_{1}\right] /$ forced vital capacity [FVC] ratio $<70 \%$ ). Multivariable logistic regression was used to identify factors associated with ICS use.

Results: A total of 901 patients were included, of which $47.9 \%(\mathrm{n}=432)$ were treated with ICS. A total of 240 patients $(26.6 \%)$ experienced moderate/severe exacerbations in the prior year, while 309 (34.3\%) during the previous two years. History of asthma totaled $11.6 \%(\mathrm{n}=105)$. The most frequent phenotype was non-exacerbator $(51.6 \%)$, and the proportion of patient with moderate or severe exacerbations was significantly higher among ICS treated patients compared to non-treated: $37.5 \%$ versus $16.6 \%$ during the previous year ( $\mathrm{p}<0.001$ ), and $46.8 \%$ versus $22.8 \%$ during the previous 2 -years $(\mathrm{p}<0.001)$, respectively. Patient characteristics were similar among spirometry confirmed patients and the overall population. Factors significantly associated with ICS use were a history of asthma $(\mathrm{OR}=4.39,95 \% \mathrm{CI}: 2.67-7.26)$, the presence of moderate or severe exacerbations in the last year $(\mathrm{OR}=2.52,95 \% \mathrm{CI}: 1.81-$ 3.49), followed by higher mMRC and higher CAT score.

Conclusion: Nearly half of patients in primary care in Spain are treated with ICS, despite most of them being non-exacerbators. History of asthma, exacerbations, and worse dyspnea and CAT scores are associated with ICS use.

Keywords: chronic obstructive pulmonary disease, primary care, inhaled corticosteroids, exacerbations

\section{Introduction}

Chronic obstructive pulmonary disease (COPD) is a significant public health problem, causing $2.6 \%$ of global disabilityadjusted life years worldwide. ${ }^{1}$ Disease prevalence increases with age, and it is estimated to affect $11.8 \%$ individuals aged 40 or above in Spain, although underdiagnosis is frequent. ${ }^{2}$

Exacerbations are the leading cause of COPD-associated mortality. ${ }^{3,4}$ In this sense, one of the main goals of pharmacological therapy is to reduce the number and severity of acute exacerbations. ${ }^{5}$ Different clinical guidelines have recognized the usefulness of inhaled corticosteroids (ICS) in patients with frequent exacerbations despite optimal bronchodilator treatment, by decreasing the number of exacerbations and improving quality of life. ${ }^{6,7}$ However, long-term use of ICS is associated with an increased risk of significant side-effects, ${ }^{8,9}$ for which ICS should be reserved for individuals at high exacerbation risk. 
Both blood eosinophils and previous history of exacerbations are the criteria defined in guidelines for the use of ICS. The Spanish COPD guidelines recommend the use of ICS for patients with frequent exacerbations and an eosinophilic phenotype. ${ }^{10}$ The Global Initiative for Chronic Obstructive Lung Disease (GOLD) 2019 strategy $^{6}$ recommends ICS for individuals in group $\mathrm{D}$ with more than 300 blood eosinophils $\mu \mathrm{L}$, and as continuation therapy in patients with frequent exacerbations despite initial therapy and more than 100 eosinophils $/ \mu \mathrm{L}$. However, discrepancies exist between guidelines and real-life practice, given that approximately two-thirds of COPD patients are non-exacerbators and ICS therapy is not recommended, although more than $60 \%$ of these patients are treated with ICS in Spain. ${ }^{11,12}$ In order to prevent unnecessary risks of side effects, the recent European Respiratory Society (ERS) guideline recommends withdrawing ICS in COPD patients with infrequent exacerbations and less than 300 eosinophils in peripheral blood. ${ }^{13}$ However, despite current recommendations, a recent Spanish study conducted in primary care showed that $54 \%$ of patients initiating triple therapy including ICS had no exacerbations in the previous year and only $34 \%$ stepped down during five years follow-up. ${ }^{14}$

The aim of our study was to describe ICS use in COPD patients and identify predictors of ICS use among COPD patients treated within primary care in Spain.

\section{Materials and Methods}

\section{Design}

This was an observational, cross-sectional, non-interventional, and multicenter study among COPD patients attending Spanish Primary Care centers. The design of the study imposed a single visit coinciding with patients' routine COPD follow-up.

\section{Site and Patient Selection}

To ensure the study sample's representativeness of the COPD population in Spain, patients were recruited from 182 primary care centers in different geographical areas according to the distribution of the overall population in this area. The number of sites/patients in each Autonomous Communities was estimated according to the COPD population distribution extracted from the Spanish National Institute of Statistics (INE).$^{15}$

To minimize selection bias at the patient level, the first 5 consecutive patients from each site who met all selection criteria and provided written informed consent prior to participation were enrolled between September 2018 and January 2020. Inclusion criteria were: individuals aged 40 or above, a COPD diagnosis of more than 2 years before the study visit, previously confirmed COPD diagnosis by post-bronchodilator forced expiratory volume in 1 second $\left(\mathrm{FEV}_{1}\right)$ /forced vital capacity $(\mathrm{FVC})$ ratio $<70 \%$, clinical records with at least a 2-year history available before the study visit, and the ability to complete COPD Assessment Test (CAT). Individuals with a moderate or severe exacerbation at the time of the study visit or within the previous 4 weeks before the study visit were excluded from the study.

\section{Data Sources and Variables}

Investigators collected socio-demographic and clinical variables directly from patients' medical records. Information included demographic variables, smoking status and pack-years, body mass index (BMI) $\left(\mathrm{Kg} / \mathrm{m}^{2}\right)$, comorbidities, history of asthma, spirometry data, blood eosinophils, time since COPD diagnosis, and COPD treatment, among others. Current ICS treatment was obtained from medical charts and was collected as current use of ICS.

Moderate and severe exacerbations occurring within the last year and last 2-years prior to the study visit were recorded. Moderate exacerbations were considered as an increase in respiratory symptoms significant enough to require additional medication with antibiotics and/or systemic corticosteroids, while severe exacerbations were those requiring hospitalization.

Blood eosinophils count was categorized into high/low, using $\geq 300$ cell $/ \mu \mathrm{L}$ as the threshold. Additionally, because many patients' eosinophil values were reported in percentage (\%), a threshold was established at the 75 percentilepoint, ${ }^{16}$ which for this cohort was equaled 3.6\% (high eosinophils $\geq 3.6 \%$, low eosinophils $<3.6 \%$ ). The body mass index, obstruction, dyspnea and exacerbations (BODEx) index, which ranges between 0-9 and computes BMI, degree of dyspnea measured with the modified Medical Research Council (mMRC) scale, pulmonary function measured with the 
$\mathrm{FEV}_{1}(\%)$ post-bronchodilation, and the number of severe exacerbations, were used to assess COPD prognosis. ${ }^{17}$ The COPD specific comorbidity test (COTE) index, which ranges between 0 and 25, was used to assess patients' comorbidities burden in COPD associated with a higher risk of death. ${ }^{18}$

At the study visit, patients completed the mMRC dyspnea scale, which ranges from 0 to 4 , where a higher score indicates higher degree of breathlessness, and the CAT, to assess the impact of COPD on symptoms and usual activities (ranges from 0 to 40) with higher scores denoting a more severe impact of COPD on patient's quality of life. All adverse events commonly related to ICS use, irrespective of when they occurred, were collected at the study visit from medical charts (pneumonia, fracture, skin thinning/easy bruising, cataracts, diabetes, oropharyngeal candidiasis).

\section{Patient Subgroups}

Patients' characteristics were classified according to the phenotypes of the Spanish guidelines for COPD 2017 (GesEPOC 2017) (non exacerbator, exacerbator with emphysema, exacerbator chronic bronchitis, asthma-COPD overlap syndrome), since this was an observational study, no computed tomography was required, and the diagnosis of emphysema was based on the clinical criteria of the investigators. ${ }^{19}$ Patients were categorized at the time of inclusion according to the GOLD 2019 patient groups (A, B, C, D), and based on their airflow limitation severity (based on post-bronchodilator $\mathrm{FEV}_{1}$ value), into four levels (I, II, III, and IV), according to the GOLD 2019 document. $^{6}$

\section{Sub-Analysis in Patients with Available Spirometry}

While all patients had a COPD diagnosis based on $\mathrm{FEV}_{1} / \mathrm{FVC}$ ratio $<70 \%$, in a significant proportion of patients' full spirometry values were not recorded in the medical chart. As a result, patients were classified according to the presence/ absence of complete spirometry values into spirometry confirmed COPD and COPD (Figure 1).

\section{Statistical Analysis}

All variables were described with measures of central tendency (mean and median), variability/dispersion (standard deviation, interquartile ranges, and ranges) for continuous variables, and distributions of absolute and relative frequencies for categorical variables. Patients were compared according to ICS use and spirometry status, using Chi-Square or Fisher's Exact test for categorical variables, and means $t$-test, Mann-Whitney $U$-test, or Kruskal Wallis test when appropriate for continuous variables.

Univariate and multivariate logistic regression models were used to assess variables associated with ICS use. Variables included in the multivariable model were selected using an explanatory approach (age, sex, spirometry (yes/ no), blood eosinophils (high/low), history of asthma (yes/no), mMRC, CAT and moderate/severe exacerbations previous year). The final model was selected using a backward elimination procedure, which selected variables with a p-value $<0.05$ in the univariate analysis. The same models were reproduced restricting the patient sample to individuals with spirometry confirmed COPD using the same procedure previously described. The $95 \%$ confidence intervals were obtained. All statistical testing was 2 -sided and a p-value $<0.05$ was used to determine statistical significance. All analyses were conducted using SAS Enterprise Guide version 7.15 software.

\section{Ethical Considerations}

The OPTI study was conducted in accordance with the protocol, the principles set forth in the Declaration of Helsinki, and the applicable Spanish regulations regarding the conduct of observational studies. All patients were required to sign the informed consent form to participate in this study. This study was approved by the Principality of Asturias' ethics committee.

\section{Results}

\section{Population}

A total of 922 patients were initially enrolled, of which 21 (2.2\%) were excluded because they did not fulfill the selection criteria, leaving 901 eligible patients (Figure 1). Mean (SD) age was 71.0 (9.8) years and 80.4\% were male. Most patients were ex-smokers (60.1\%). The mean (SD) postbronchodilator $\mathrm{FEV}_{1}(\%)$ was $65.9 \%$ (19.8\%), and high blood eosinophils 


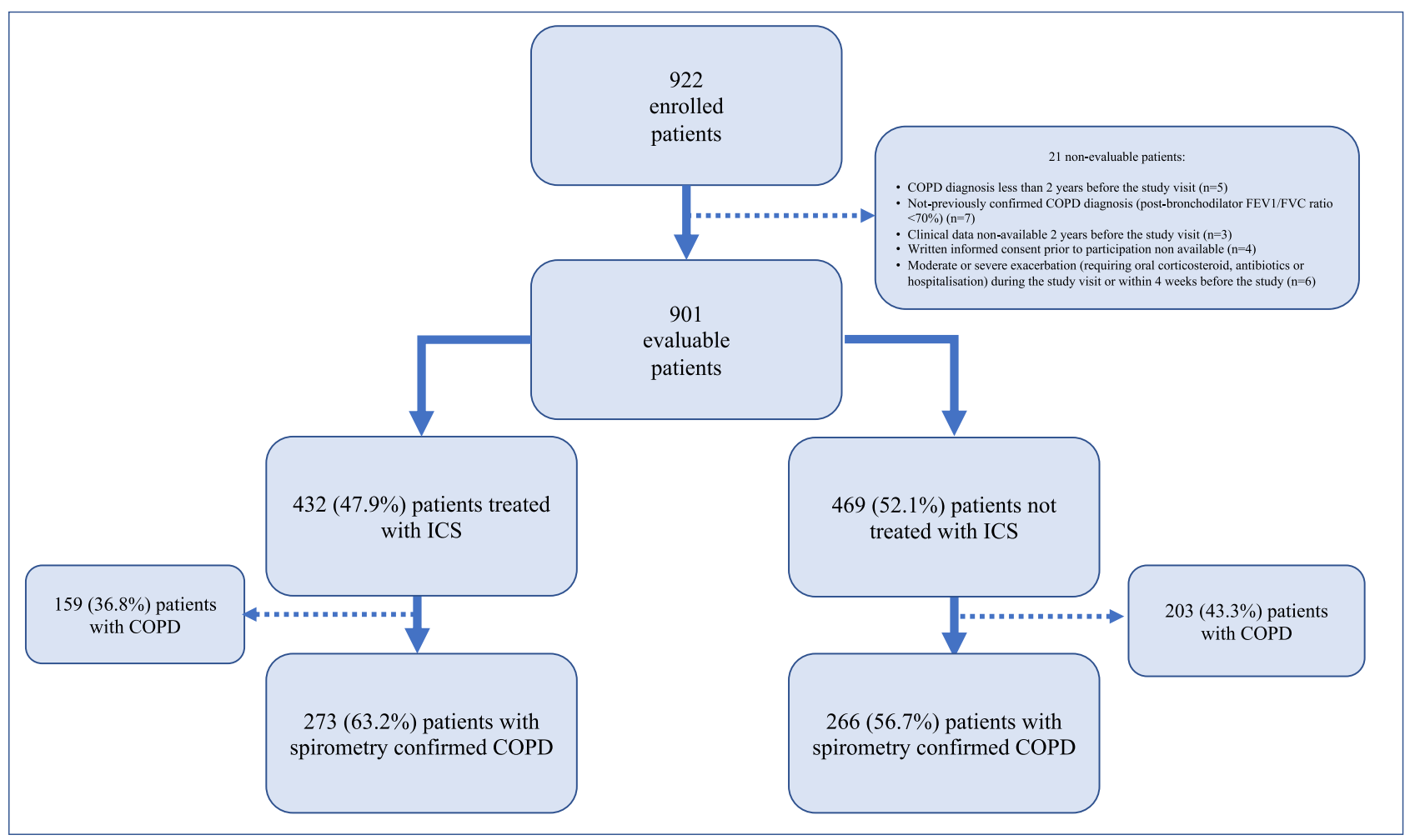

Figure I Flow chart of the study.

Note: A patient may have more than one reason.

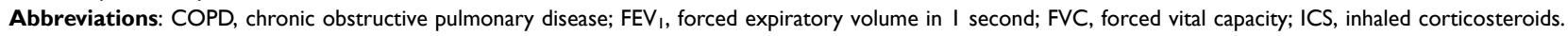

were present in $28 \%$. In terms of the GesEPOC phenotypes, most patients $(51.6 \%)$ were non-exacerbators, followed by exacerbators-chronic bronchitis type (22.5\%). The most prevalent GOLD (2019) group was group B (61.5\%), followed by $\mathrm{D}(27.2 \%)$. The proportion of patients with moderate or severe exacerbations in the last year was $26.6 \%$ and in the last 2 years $34.3 \%$. Up to $47.9 \%$ of patients were treated with ICS.

Of the 901 patients included in the study, 539 (59.8\%) patients had a spirometry confirmed COPD diagnosis. Baseline characteristics were compared among patients with spirometry confirmed COPD and COPD (Table 1), being the most remarkable differences between groups in terms of the BODEx index, the number of exacerbations in the last 2-years, and an ICS-use, which were all higher among the spirometry confirmed COPD group.

A total of 28 patients (3.1\%) experienced an ICS-related adverse event (14 patients experienced these events within two years before the study visit (1.6\%), while the rest occurred beyond that cut-off point). The most frequent events were oropharyngeal candidiasis (10 patients), pneumonia (8 patients), diabetes (7 patients), fracture (4 patients), and cataracts (3 patients). No cases of skin thinning/easy bruising were reported.

\section{Patients' Characteristics According to the Use of ICS}

For the whole group, ICS-treated patients were older, showed a lower proportion of active smokers, a greater evidence of obstruction in spirometry, a greater proportion of asthma, a greater impact of COPD measured by BODEx and mMRC, and significantly different distribution in terms of patient phenotypes and GOLD groups, with a lower proportion of nonexacerbators and a greater proportion of GOLD groups C and D when compared to non-ICS treated patients (Table 2).

\section{History of Exacerbations in Patients Treated and Not Treated with ICS}

The proportion of patient with moderate or severe exacerbations was significantly higher among ICS treated patients compared to those not treated with ICS: $37.5 \%$ versus $16.6 \%$ during the previous year $(\mathrm{p}<0.001)$, and $46.8 \%$ versus $22.8 \%$ during the previous 2 -years $(\mathrm{p}<0.001)$, respectively. The mean (SD) number of exacerbations was significantly 
Table I Characteristics of Patients Included in the Study and Comparisons Between Those with COPD and Spirometry Confirmed COPD

\begin{tabular}{|c|c|c|c|c|}
\hline Characteristics & $\begin{array}{c}\text { Total } \\
(n=901)\end{array}$ & $\begin{array}{l}\text { Spirometry Confirmed COPD } \\
\qquad(n=539)\end{array}$ & $\begin{array}{l}\text { COPD } \\
(n=362)\end{array}$ & $P$ value \\
\hline Age, years & $71.0(9.8)$ & 7I.I (9.7) & $70.8(10.0)$ & 0.731 \\
\hline Sex, male, n (\%) & $724(80.4 \%)$ & 437 (81.1\%) & 287 (79.3\%) & 0.506 \\
\hline BMI $\left(\mathrm{Kg} / \mathrm{m}^{2}\right)$ & $28.4(5.2)$ & $28.0(5.4)$ & $29.0(4.8)$ & 0.004 \\
\hline \multicolumn{5}{|l|}{ Smoking habits, n (\%) } \\
\hline Smoker & $281(31.3 \%)$ & $173(32.2 \%)$ & $108(29.9 \%)$ & 0.107 \\
\hline Ex-smoker & $540(60.1 \%)$ & $327(60.8 \%)$ & $213(59.0 \%)$ & \\
\hline Never smoker & 78 (8.7\%) & 38 (7.1\%) & $40(11.1 \%)$ & \\
\hline Pack-years & $45.4(26.7)$ & $46.4(27.2)$ & $43.6(26.0)$ & 0.193 \\
\hline \multicolumn{5}{|l|}{ Post-BD spirometry: } \\
\hline FVC, L & $3.0(0.9)$ & $3.0(0.9)$ & $2.9(0.9)$ & 0.228 \\
\hline FVC (\%) & $80.9(19.3)$ & $80.6(19.7)$ & $82.7(17.0)$ & 0.312 \\
\hline $\mathrm{FEV}_{1}, \mathrm{~L}$ & I.8 (0.7) & I.8 (0.7) & $2.1(0.7)$ & $<0.001$ \\
\hline FEV,$(\%)^{* *}$ & $65.9(19.8)$ & $63.7(19.3)$ & $76.3(18.7)$ & $<0.001$ \\
\hline $\mathrm{FEV}_{1} / \mathrm{FVC}(\%)^{* * *}$ & $6 I .1(11.4)$ & $58.0(9.3)$ & $77.2(7.2)$ & $<0.001$ \\
\hline Blood eosinophils, cells $/ \mathrm{mm}^{3}$ & $250(153)$ & $242(152)$ & $275(158)$ & 0.364 \\
\hline Blood eosinophils (\%) & $2.7(1.7)$ & $2.8(1.8)$ & $2.5(1.6)$ & 0.163 \\
\hline High blood eosinophils*, n (\%) & $175(28.0 \%)$ & $119(28.6 \%)$ & $56(26.8 \%)$ & 0.634 \\
\hline History of asthma, n (\%) & $105(11.7 \%)$ & $63(11.7 \%)$ & $42(11.6 \%)$ & 0.969 \\
\hline $\mathrm{mMRC}$ & $1.5(1.0)$ & $1.5(1.0)$ & I.5 (I.0) & 0.544 \\
\hline CAT & $14.5(7.8)$ & $14.6(7.9)$ & $14.2(7.7)$ & 0.485 \\
\hline BODEx index & $1.5(1.5)$ & $1.6(1.6)$ & $\mathrm{I} .0(\mathrm{I} . \mathrm{I})$ & $<0.001$ \\
\hline COTE index & $2.2(2.9)$ & $2.3(3.0)$ & $2.03(2.7)$ & 0.314 \\
\hline \multicolumn{5}{|l|}{ Phenotypes, n (\%) } \\
\hline Non exacerbator & $446(51.6 \%)$ & $257(49.2 \%)$ & $189(55.1 \%)$ & 0.234 \\
\hline Exacerbator with emphysema & $84(9.7 \%)$ & $57(10.9 \%)$ & 27 (7.9\%) & \\
\hline Exacerbator with chronic bronchitis & $195(22.5 \%)$ & II 8 (22.6\%) & 77 (22.4\%) & \\
\hline AcOS & 140 (16.2\%) & $90(17.2 \%)$ & $50(14.6 \%)$ & \\
\hline \multicolumn{5}{|l|}{ GOLD group, n (\%) } \\
\hline A & $93(10.3 \%)$ & $54(10.0 \%)$ & $39(10.8 \%)$ & 0.962 \\
\hline B & $554(61.5 \%)$ & 331 (6I.4\%) & $223(61.6 \%)$ & \\
\hline C & $9(1.0 \%)$ & $5(0.9 \%)$ & $4(1.1 \%)$ & \\
\hline D & $245(27.2 \%)$ & 149 (27.6\%) & $96(26.5 \%)$ & \\
\hline \multicolumn{5}{|l|}{ Exacerbations previous year: } \\
\hline Moderate, n (\%) & $186(20.6 \%)$ & $119(22.1 \%)$ & $67(\mid 8.5 \%)$ & 0.194 \\
\hline $\begin{array}{l}\text { Mean number of moderate exacerbations in the last year } \\
\text { per patient (all) }\end{array}$ & $0.4(0.9)$ & $0.4(1.0)$ & $0.3(0.9)$ & 0.211 \\
\hline
\end{tabular}


Table I (Continued).

\begin{tabular}{|c|c|c|c|c|}
\hline Characteristics & $\begin{array}{c}\text { Total } \\
(\mathrm{n}=901)\end{array}$ & $\begin{array}{l}\text { Spirometry Confirmed COPD } \\
\qquad(n=539)\end{array}$ & $\begin{array}{c}\text { COPD } \\
(n=362)\end{array}$ & $P$ value \\
\hline Severe, $\mathrm{n}(\%)$ & 77 (8.5\%) & $52(9.6 \%)$ & 25 (6.9\%) & 0.149 \\
\hline $\begin{array}{l}\text { Mean number of severe exacerbations in the last year per } \\
\text { patient (all) }\end{array}$ & $0.1(0.4)$ & $0.1(0.5)$ & $0.1(0.3)$ & 0.144 \\
\hline Moderate/severe, n (\%) & $240(26.6 \%)$ & $152(28.2 \%)$ & $88(24.3 \%)$ & 0.195 \\
\hline Mean number of moderate/severe (all) & $0.5(1.0)$ & $0.5(1.1)$ & $0.4(0.9)$ & 0.086 \\
\hline \multicolumn{5}{|l|}{ Exacerbations in the previous 2 years: } \\
\hline Moderate/severe, n (\%) & $309(34.3 \%)$ & $196(36.4 \%)$ & $113(31.2 \%)$ & 0.111 \\
\hline Number of moderate/severe (all) & $0.8(1.7)$ & $0.9(1.8)$ & $0.7(1.5)$ & 0.047 \\
\hline \multicolumn{5}{|l|}{ Treatment, n (\%) } \\
\hline None & $46(5.1 \%)$ & $21(3.9 \%)$ & $25(6.9 \%)$ & 0.033 \\
\hline LAMA or LABA & $96(10.7 \%)$ & 62 (11.5\%) & $34(9.4 \%)$ & \\
\hline LAMA/LABA & $316(35.1 \%)$ & 175 (32.5\%) & $|4|(39.0 \%)$ & \\
\hline LAMA/ICS or LABA/ICS & $110(12.2 \%)$ & $63(11.7 \%)$ & 47 (13.0\%) & \\
\hline LAMA/LABA/ICS & $317(35.2 \%)$ & 207 (38.4\%) & $110(30.4 \%)$ & \\
\hline Other & $16(1.8 \%)$ & II (2.0\%) & 5 (I.4\%) & \\
\hline ICS (any), n (\%) & $432(47.9 \%)$ & 273 (50.6\%) & $159(43.9 \%)$ & 0.048 \\
\hline
\end{tabular}

Notes: Values are means (SD), unless otherwise specified; *high eosinophils are defined as $\geq 3.6 \%$ or $\geq 300$ cell/ $\mu \mathrm{L}$ and valid $\mathrm{n}=625$, of which 416 are spirometry confirmed; **FEV $\%$ valid $n=633 ; * * * F E V_{1} / F V C \%$, valid $n=643$.

Abbreviations: ACOS, asthma COPD overlap syndrome; BD, bronchodilator; BMI, body mass index; BODEx, body mass index, obstruction, dyspnea and exacerbations index; CAT, COPD assessment test; COPD, chronic obstructive pulmonary disease; COTE, COPD comorbidity test; FEV , forced expiratory volume in I second; FVC, forced vital capacity; ICS, inhaled corticosteroids; LABA, long-acting beta2-agonist; LAMA, long-acting muscarinic antagonists; mMRC, modified Medical Research Council; SD, standard deviation.

higher among ICS treated patients, at 0.72 (1.23) exacerbations in the prior year and 1.27 (1.99) exacerbations in the last two years $(\mathrm{p}>0.001)$ (Table 3$)$.

Among the 432 patients treated with ICS, $70.4 \%$ had eosinophil counts $(68.4 \%$ low and $31.6 \%$ high eosinophils). Patients without exacerbations in the prior year totaled $38.5 \%$ in the high eosinophil count group and $33.6 \%$ in the low eosinophil count group. The majority of ICS treated patients without exacerbations in the previous year did not experience any exacerbation during the previous two years, 56.7\% among the high eosinophil count group and $61.4 \%$ among the low eosinophil count group (Figure 2A). The distribution of eosinophil counts and exacerbations in patients without ICS treatment is shown in Figure 2B.

\section{ICS Use According to Different Guidelines and Patient Scales}

ICS use varied among the different phenotypes, with the highest proportion of patients treated with ICS among those with an asthma COPD overlap syndrome (ACOS) phenotype (81.4\%), followed by the exacerbator with emphysema phenotype (63.1\%) (Figure 3A). ICS use according to the GOLD spirometric classification was below $50 \%$ for groups I and II, while $64.1 \%$ and $68.8 \%$ for groups III and IV, respectively (Figure 4B). Among the GOLD 2019 patient groups, groups A$\mathrm{C}$ ranged between $33 \%$ and $42 \%$, while group D had a higher proportion of ICS treated patients, at $67.8 \%$. Patients treated with ICS showed a greater impact of COPD measured by all patient scales (BODEx, CAT, and mMRC) (Figure 3B).

\section{Factors Independently Associated with the Prescription of ICS for COPD in Primary Care}

Overall, individuals with a prior history of asthma were more likely to be treated with ICS than those without asthma (Figure 4A) $(\mathrm{OR}=4.39,95 \% \mathrm{CI}$ : 2.67-7.26). Similarly, patients with moderate or severe exacerbations in the last year 
Table 2 Comparison of Characteristics Between Patients Treated or Not Treated with ICS in Patients with COPD and in Those with Spirometry Confirmed COPD

\begin{tabular}{|c|c|c|c|c|c|c|}
\hline \multirow[t]{2}{*}{ Characteristics } & \multicolumn{3}{|c|}{ Spirometry Confirmed COPD $(n=539)$} & \multicolumn{3}{|c|}{ COPD $(n=362)$} \\
\hline & $\begin{array}{l}\text { Treated with } \\
\text { ICS }(n=273)\end{array}$ & $\begin{array}{l}\text { Not Treated with } \\
\text { ICS }(n=266)\end{array}$ & P value & $\begin{array}{l}\text { Treated with } \\
\text { ICS }(n=159)\end{array}$ & $\begin{array}{l}\text { Not Treated with } \\
\text { ICS }(n=203)\end{array}$ & $P$ value \\
\hline Age, years & $72.0(9.9)$ & $70.1(9.4)$ & 0.023 & $70.5(10.9)$ & $71.1(9.3)$ & 0.539 \\
\hline Sex, male, n (\%) & $220(80.6 \%)$ & $217(81.6 \%)$ & 0.769 & $123(77.4 \%)$ & $164(80.8 \%)$ & 0.424 \\
\hline BMI $\left(\mathrm{Kg} / \mathrm{m}^{2}\right)$ & $27.8(5.2)$ & $28.1(5.5)$ & $0.48 \mathrm{I}$ & $29.4(5.2)$ & $28.7(4.5)$ & 0.156 \\
\hline $\begin{array}{l}\text { Smoking habits, n (\%) } \\
\text { Smoker } \\
\text { Ex-smoker } \\
\text { Never smoker }\end{array}$ & $\begin{array}{c}74(27.2 \%) \\
176(64.7 \%) \\
22(8.1 \%)\end{array}$ & $\begin{array}{c}99(37.2 \%) \\
|5|(56.8 \%) \\
16(6.0 \%)\end{array}$ & 0.041 & $\begin{array}{c}50(31.6 \%) \\
94(59.5 \%) \\
14(8.9 \%)\end{array}$ & $\begin{array}{l}58(28.6 \%) \\
119(58.6 \%) \\
26(12.8 \%)\end{array}$ & 0.463 \\
\hline Pack-years & $46.5(27.8)$ & $46.4(26.6)$ & 0.985 & $43.3(23.5)$ & $43.8(27.8)$ & 0.889 \\
\hline Post-BD spirometry & & & & & & \\
\hline FVC, L & $2.8(0.9)$ & $3.2(1.0)$ & $<0.001$ & $3.0(0.8)$ & $2.8(0.9)$ & 0.440 \\
\hline FVC (\%) & $77.9(18.8)$ & $83.2(20.2)$ & 0.002 & $82.1(18.3)$ & $83.0(16.2)$ & 0.789 \\
\hline $\mathrm{FEV}_{1}, \mathrm{~L}$ & $1.6(0.6)$ & $1.9(0.7)$ & $<0.001$ & $2.2(0.7)$ & $2.1(0.6)$ & 0.395 \\
\hline $\mathrm{FEV}_{\mathrm{I}}(\%)$ & $60.3(19.5)$ & $67.1(18.4)$ & $<0.001$ & $75.2(21.0)$ & $77.1(17.2)$ & 0.596 \\
\hline $\mathrm{FEV}_{1} / \mathrm{FVC}$ & $56.8(9.9)$ & $59.2(8.5)$ & 0.002 & $77.7(7.3)$ & $76.9(7.1)$ & 0.560 \\
\hline $\begin{array}{l}\text { Blood eosinophils, cells/ } \\
\mathrm{mm}^{3} \text { (corrected) }\end{array}$ & $234(152)$ & $249(153)$ & 0.676 & $283(164)$ & $26 \mid(156)$ & 0.750 \\
\hline Blood eosinophils (\%) & $2.8(1.9)$ & $2.7(1.6)$ & 0.418 & $2.5(1.6)$ & $2.5(1.6)$ & 0.999 \\
\hline $\begin{array}{l}\text { High blood eosinophils*, } \\
\text { n (\%) }\end{array}$ & 65 (31.9\%) & $54(25.5 \%)$ & 0.149 & $28(28.9 \%)$ & $28(25.0 \%)$ & 0.529 \\
\hline History of asthma, n (\%) & $47(17.2 \%)$ & $16(6.0 \%)$ & $<0.001$ & $35(22.0 \%)$ & $7(3.4 \%)$ & $<0.001$ \\
\hline mMRC & $1.8(1.1)$ & $1.2(0.9)$ & $<0.001$ & $1.6(1.1)$ & $1.3(0.9)$ & 0.015 \\
\hline CAT & $16.2(8.1)$ & $12.9(7.3)$ & $<0.001$ & $15.8(7.9)$ & I3.0 (7.3) & 0.001 \\
\hline BODEx index & $2.0(1.8)$ & $1.2(1.3)$ & $<0.001$ & $1.2(1.3)$ & $0.8(0.9)$ & 0.082 \\
\hline COTE index & $2.4(3.1)$ & $2.2(2.9)$ & 0.520 & $2.4(2.8)$ & I.8 (2.6) & 0.020 \\
\hline Phenotypes, n (\%) & & & & & & \\
\hline $\begin{array}{l}\text { Non exacerbator } \\
\text { Exacerbator with } \\
\text { emphysema } \\
\text { Exacerbator with chronic } \\
\text { bronchitis } \\
\text { ACOS }\end{array}$ & $\begin{array}{l}71(27.1 \%) \\
40(15.3 \%) \\
80(30.5 \%) \\
71(27.1 \%)\end{array}$ & $\begin{array}{l}186(71.5 \%) \\
17(6.5 \%) \\
38(14.6 \%) \\
19(7.3 \%)\end{array}$ & $<0.001$ & $\begin{array}{l}57(38.5 \%) \\
13(8.8 \%) \\
35(23.6 \%) \\
43(29.1 \%)\end{array}$ & $\begin{array}{l}132(67.7 \%) \\
14(7.2 \%) \\
42(21.5 \%) \\
7(3.6 \%)\end{array}$ & $<0.001$ \\
\hline $\begin{array}{l}\text { GOLD group, } n(\%) \\
\text { A } \\
\text { B } \\
\text { C } \\
\text { D }\end{array}$ & $\begin{array}{c}2 \mid(7.7 \%) \\
140(51.3 \%) \\
\text { I }(0.4 \%) \\
|| \mid(40.7 \%)\end{array}$ & $\begin{array}{c}33(12.4 \%) \\
19 \mid(71.8 \%) \\
4(1.5 \%) \\
38(14.3 \%)\end{array}$ & $<0.001$ & $\begin{array}{c}18(11.3 \%) \\
84(52.8 \%) \\
2(1.3 \%) \\
55(34.6 \%)\end{array}$ & $\begin{array}{c}21(10.3 \%) \\
139(68.5 \%) \\
2(1.0 \%) \\
4 \mid(20.2 \%)\end{array}$ & 0.014 \\
\hline
\end{tabular}


Table 2 (Continued).

\begin{tabular}{|c|c|c|c|c|c|c|}
\hline \multirow[t]{2}{*}{ Characteristics } & \multicolumn{3}{|c|}{ Spirometry Confirmed COPD $(n=539)$} & \multicolumn{3}{|c|}{ COPD $(n=362)$} \\
\hline & $\begin{array}{l}\text { Treated with } \\
\text { ICS }(n=273)\end{array}$ & $\begin{array}{l}\text { Not Treated with } \\
\text { ICS }(n=266)\end{array}$ & $P$ value & $\begin{array}{l}\text { Treated with } \\
\text { ICS }(n=159)\end{array}$ & $\begin{array}{l}\text { Not Treated with } \\
\text { ICS }(n=203)\end{array}$ & $P$ value \\
\hline \multicolumn{7}{|l|}{ Exacerbations previous year } \\
\hline Moderate, $\mathrm{n}(\%)$ & $83(30.4 \%)$ & $36(13.5 \%)$ & $<0.001$ & $42(26.4 \%)$ & $25(12.3 \%)$ & 0.001 \\
\hline Mean number of moderate & $0.6(1.2)$ & $0.2(0.8)$ & $<0.001$ & $0.5(\mathrm{I} . \mathrm{I})$ & $0.2(0.7)$ & 0.001 \\
\hline Severe, $n(\%)$ & $4 \mathrm{I}(15.0 \%)$ & II (4.I\%) & $<0.001$ & $16(10.1 \%)$ & 9 (4.4\%) & 0.036 \\
\hline Mean number of severe & $0.2(0.6)$ & $0.1(0.3)$ & 0.002 & $0.1(0.5)$ & $0.0(0.2)$ & 0.014 \\
\hline Moderate/severe, $n(\%)$ & $107(39.2 \%)$ & 45 (16.9\%) & $<0.001$ & 55 (34.6\%) & $33(16.3 \%)$ & $<0.001$ \\
\hline $\begin{array}{l}\text { Mean number of moderate/ } \\
\text { severe (all) }\end{array}$ & $0.8(1.3)$ & $0.3(0.8)$ & $<0.001$ & $0.6(1.1)$ & $0.2(0.7)$ & $<0.001$ \\
\hline \multicolumn{7}{|l|}{$\begin{array}{l}\text { Exacerbations in the } \\
\text { previous } 2 \text { years }\end{array}$} \\
\hline Moderate/severe, $n(\%)$ & 137 (50.2\%) & 59 (22.2\%) & $<0.001$ & 65 (40.9\%) & 48 (23.6\%) & $<0.001$ \\
\hline $\begin{array}{l}\text { Number of moderate/ } \\
\text { severe }\end{array}$ & $1.4(2.1)$ & $0.5(1.3)$ & $<0.001$ & I.I (I.8) & $0.4(1.2)$ & $<0.001$ \\
\hline \multicolumn{7}{|l|}{ Treatment, n (\%) } \\
\hline None & 21 (7.9\%) & - & $<0.001$ & - & $25(12.3 \%)$ & $<0.001$ \\
\hline LAMA or LABA & $62(23.3 \%)$ & - & & - & $34(16.7 \%)$ & \\
\hline LAMA/LABA & 175 (65.8\%) & - & & - & $|4|$ (69.5\%) & \\
\hline LAMA/ICS or LABA/ICS & - & $63(23.1 \%)$ & & 47 (29.6\%) & - & \\
\hline LAMA/LABA/ICS & - & 207 (75.8\%) & & 110 (69.2\%) & - & \\
\hline Other & $8(3.0 \%)$ & $3(1.1 \%)$ & & $2(1.3 \%)$ & $3(1.5 \%)$ & \\
\hline ICS (any), n (\%) & $273(100 \%)$ & - & - & $159(100 \%)$ & - & - \\
\hline
\end{tabular}

Notes: Values are means (SD), unless otherwise specified; *high eosinophils are defined as $\geq 3.6 \%$ or $\geq 300$ cell/ $\mu \mathrm{L}$ and valid $\mathrm{n}=4 \mathrm{l} 6$ among spirometry confirmed patients, of which 204 were treated with ICS; and $n=209$ for COPD patients, of which 97 were treated with ICS.

Abbreviations: ACOS, asthma COPD overlap syndrome; BD, bronchodilator; BMI, body mass index; BODEx, body mass index, obstruction, dyspnea and exacerbations index; CAT, COPD assessment test; COPD, chronic obstructive pulmonary disease; COTE, COPD comorbidity test; FEV , forced expiratory volume in I second; FVC, forced vital capacity; ICS, inhaled corticosteroids; LABA, long-acting beta2-agonist; LAMA, long-acting muscarinic antagonists; mMRC, modified Medical Research Council; SD, standard deviation.

were also more likely to be treated with ICS, evidenced by an OR $=2.52$ (95\% CI: $1.81-3.49)$. The increase in each grade of mMRC and each unit in CAT were both associated with an increased use of ICS, being the OR for each 2 grades increase in mMRC of 1.58 (95\% CI: 1.10-2.27), and for each 5 units increase in CAT 1.14 (95\% CI: 1.01-1.29).

\section{Spirometry Confirmed COPD Sub-Analysis}

A total of 539 subjects had spirometry confirmed COPD, and 50.6\% were treated with ICS. ICS treated patients were generally older, more likely to be ex-smokers, and spirometry parameters were generally lower compared to non-ICS treated COPD confirmed patients (Table 2). ICS treated patients more frequently had a history of asthma and a higher symptom severity as evidenced by a higher mMRC. ICS-treated patients were mostly exacerbators with chronic bronchitis $30.5 \%$, or non-exacerbators $27.1 \%$, or ACOS totaled $27.1 \%$, while non-ICS treated were predominantly nonexacerbator types (71.5\%). In terms of GOLD 2019 groups, ICS treated patients were mostly classified as group B (51.3\%) or group D (40.7\%), while non-ICS treated were mostly group B (71.8\%). Exacerbations, both in the year and 2-years prior to the study visit were more frequent among those treated with ICS. 
Table 3 Previous Exacerbations in Patients with and without ICS Treatment

\begin{tabular}{|c|c|c|c|c|c|c|}
\hline \multirow[t]{2}{*}{ Characteristics } & \multicolumn{3}{|c|}{ Total $(n=901)$} & \multicolumn{3}{|c|}{ Spirometry Confirmed COPD $(n=539)$} \\
\hline & $\begin{array}{l}\text { Treated with ICS } \\
\qquad(n=432)\end{array}$ & $\begin{array}{l}\text { Not Treated with ICS } \\
\qquad(n=469)\end{array}$ & $P$ value & $\begin{array}{l}\text { Treated with ICS } \\
(n=273)\end{array}$ & $\begin{array}{l}\text { Not Treated with ICS } \\
\qquad(n=266)\end{array}$ & $P$ value \\
\hline \multicolumn{7}{|l|}{ Exacerbations previous year: } \\
\hline Moderate, n(\%) & $125(28.9 \%)$ & $61(13.0 \%)$ & $<0.001$ & $83(30.4 \%)$ & $36(13.5 \%)$ & $<0.001$ \\
\hline Moderate, mean (SD) (all) & $0.55(1.12)$ & $0.22(0.7 I)$ & $<0.001$ & $0.58(1.15)$ & $0.23(0.76)$ & $<0.001$ \\
\hline Severe, $n(\%)$ & $57(13.2 \%)$ & $20(4.3 \%)$ & $<0.001$ & $4 \mid(15.0 \%)$ & II (4.1\%) & $<0.001$ \\
\hline Severe, mean (SD) (all) & $0.17(0.54)$ & $0.05(0.24)$ & $<0.001$ & $0.20(0.59)$ & $0.05(0.26)$ & $<0.001$ \\
\hline Moderate/severe, $\mathrm{n}(\%)$ & $162(37.5 \%)$ & $78(16.6 \%)$ & $<0.001$ & $107(39.2 \%)$ & $45(16.9 \%)$ & $<0.001$ \\
\hline $\begin{array}{l}\text { Moderate/severe, mean } \\
\text { (SD) (all) }\end{array}$ & $0.72(1.23)$ & $0.26(0.77)$ & $<0.001$ & $0.77(1.29)$ & $0.28(0.8 \mathrm{I})$ & $<0.001$ \\
\hline \multicolumn{7}{|c|}{ Exacerbations in the previous 2 years: } \\
\hline Moderate/severe, $n(\%)$ & $202(46.8 \%)$ & $107(22.8 \%)$ & $<0.001$ & 137 (50.2\%) & $59(22.2 \%)$ & $<0.001$ \\
\hline $\begin{array}{l}\text { Moderate/severe, mean } \\
\text { (SD) (all) }\end{array}$ & $1.27(1.99)$ & $0.43(1.25)$ & $<0.001$ & $1.40(2.09)$ & $0.45(1.31)$ & $<0.001$ \\
\hline
\end{tabular}

Abbreviations: COPD, chronic obstructive pulmonary disease; ICS, inhaled corticosteroids; SD, standard deviation.

Factors significantly associated with ICS use were similar to those identified for the overall population, except for CAT which lost its statistical significance, while mMRC increased its effect size (OR $=1.43$ for each grade increase, $95 \%$ CI: 1.17-1.74), and $\mathrm{FEV}_{1} \%$, which was added to this model, exhibited per each 10 units decrease an OR for ICS use of 1.13 (95\% CI: 1.02-1.25) (Figure 4B).

\section{Discussion}

This study describes ICS-use in a nationwide COPD cohort of patients treated within primary care in terms of prior episodes of COPD exacerbations, eosinophil levels, and patient guidelines classifications. Of the 901 patients analyzed,

A

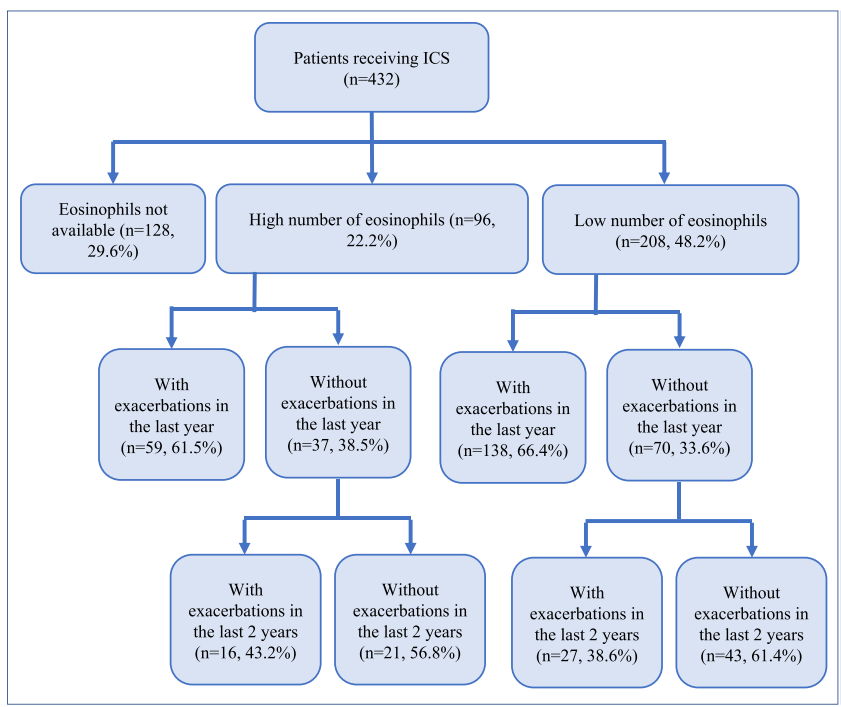

B

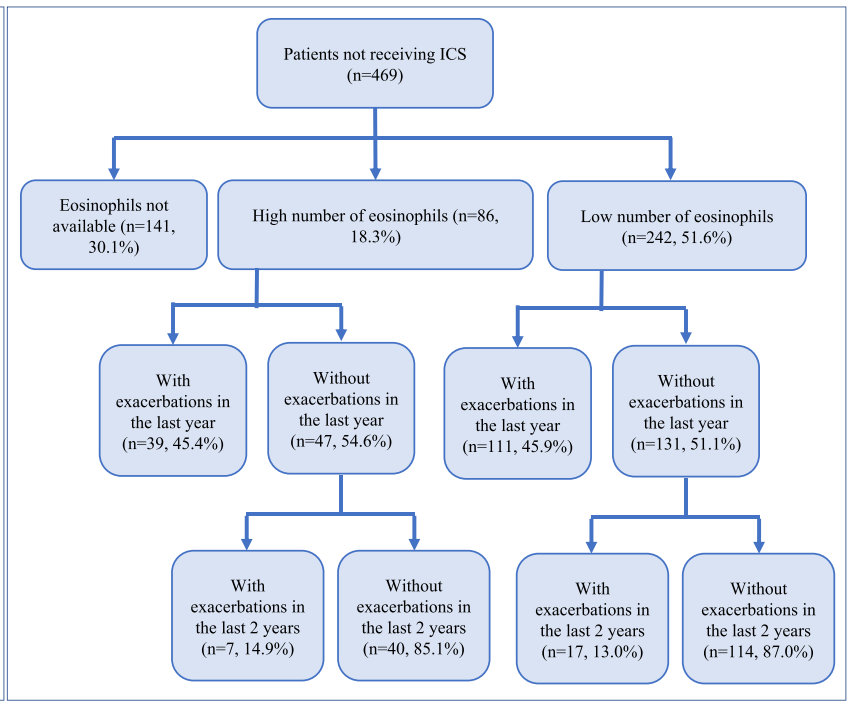

Figure 2 Distribution of the population according to the blood eosinophil levels and the frequency of exacerbations for: (A) patients on ICS; (B) patients not on ICS. Notes: Low number of eosinophils ( $<300$ cell $/ \mu \mathrm{L}$ or $<3.6 \%$ ); high number of eosinophils $(\geq 3.6 \%$ or $\geq 300$ cell $/ \mu \mathrm{L})$. Percentage totals are based on the previous row's total. Abbreviation: ICS, inhaled corticosteroids. 


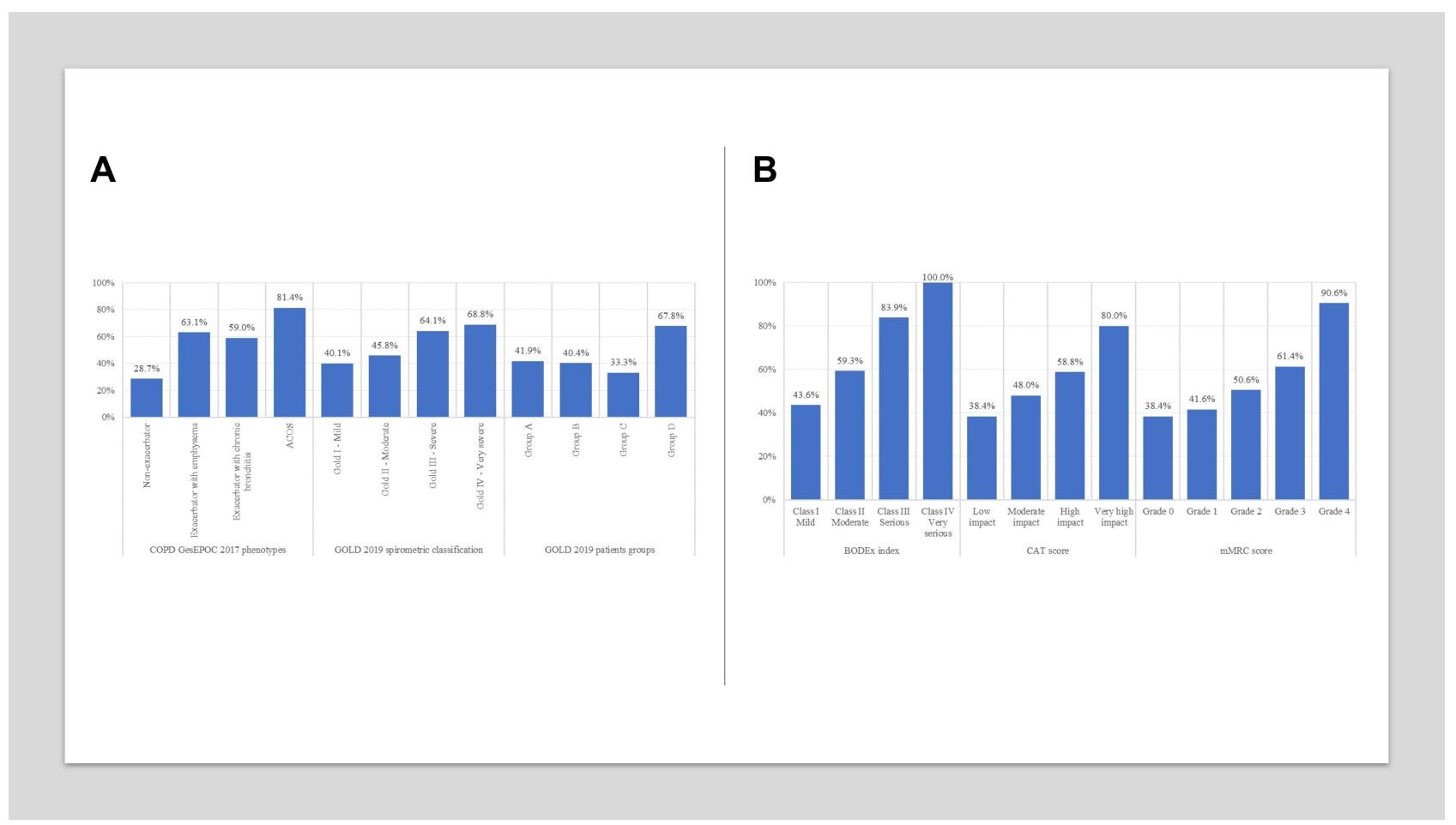

Figure 3 Use of ICS according to (A) the GOLD groups and GESEPOC level of risk and phenotypes, and (B) patients' scales.

Abbreviations: ACOS, asthma COPD overlap syndrome; BODEx, body mass index, obstruction, dyspnea and exacerbations index; CAT, COPD assessment test; COPD, chronic obstructive pulmonary disease; GOLD, Global Initiative for Chronic Obstructive Lung Disease; mMRC, modified Medical Research Council Dyspnea Scale.

patient characteristics among spirometry or clinically confirmed COPD diagnosis were similar. Up to $47.9 \%$ of the patients included were treated with ICS, while only $26.6 \%$ patients experienced moderate/severe exacerbations in the previous year. Factors associated with ICS use were having a prior history of asthma, presence of moderate or severe exacerbations in the last year, and higher $\mathrm{mMRC}$ and CAT scores. These findings were similar among those with spirometry confirmed COPD.

ICS use within our sample was high, at $47.9 \%$. This is in line with previous studies analyzing ICS use in primary care in different countries, in which ICS use ranged between $50 \%$ and $65 \%$ and a high overuse was noted. ${ }^{20-22}$ Prescribing ICS only when recommended is relevant to avoid the potential risk of side effects, since long-term use of ICS has showed a higher prevalence and incidence of serious side effects such as pneumonia, osteoporosis, diabetes, and others, beyond increasing healthcare expenditure among COPD patients. ${ }^{8,9,23}$

Several guidelines have recognized the need to withdraw ICS in patients without indications. ${ }^{10,13,24,25}$ However, a considerable proportion of patients continue receiving ICS despite not having a clear indication. In this sense, while treatment may have or have not been initially warranted at the time of prescription, clinicians should be reminded to review ICS use and step down unnecessary treatments, especially in patients with eosinophil counts $<300$ cells $/ \mu \mathrm{L}$ and less than 2 exacerbations per year without hospitalizations. ${ }^{13}$

Factors associated with ICS use within our sample were a positive history of asthma, presence of moderate/severe exacerbation in the last year and increasing mMRC and CAT scores. In a previous observational study by our research group, the results were similar to those presented in OPTI: 15,440 patients were included (70\% men, mean age 68.6 years), and $44.6 \%$ were prescribed ICS. The largest association with ICS prescription was asthma comorbidity (OR: 3.50 ; 95\% CI: 3.12-3.92), followed by exacerbation history (OR: 2.23; 95\% CI: 2.07-2.47). In addition, smoking status, spirometry, atopic dermatitis, allergic rhinitis and mean age were significantly $(\mathrm{p}<0.001)$ associated with ICS treatment. In the spirometry-confirmed population, asthma (OR: 2.89; 95\% CI: 2.29-3.64) and exacerbations were also the major factors (OR: 2.85; 95\% CI: 2.45-3.32) followed by severe bronchial-obstruction (OR: 2.63; 95\% CI: 2.24-3.08). Highdose ICS prescription was mainly associated with severe obstruction (OR: 2.27 ; 95\% CI: 1.93-2.68). ${ }^{23}$ 


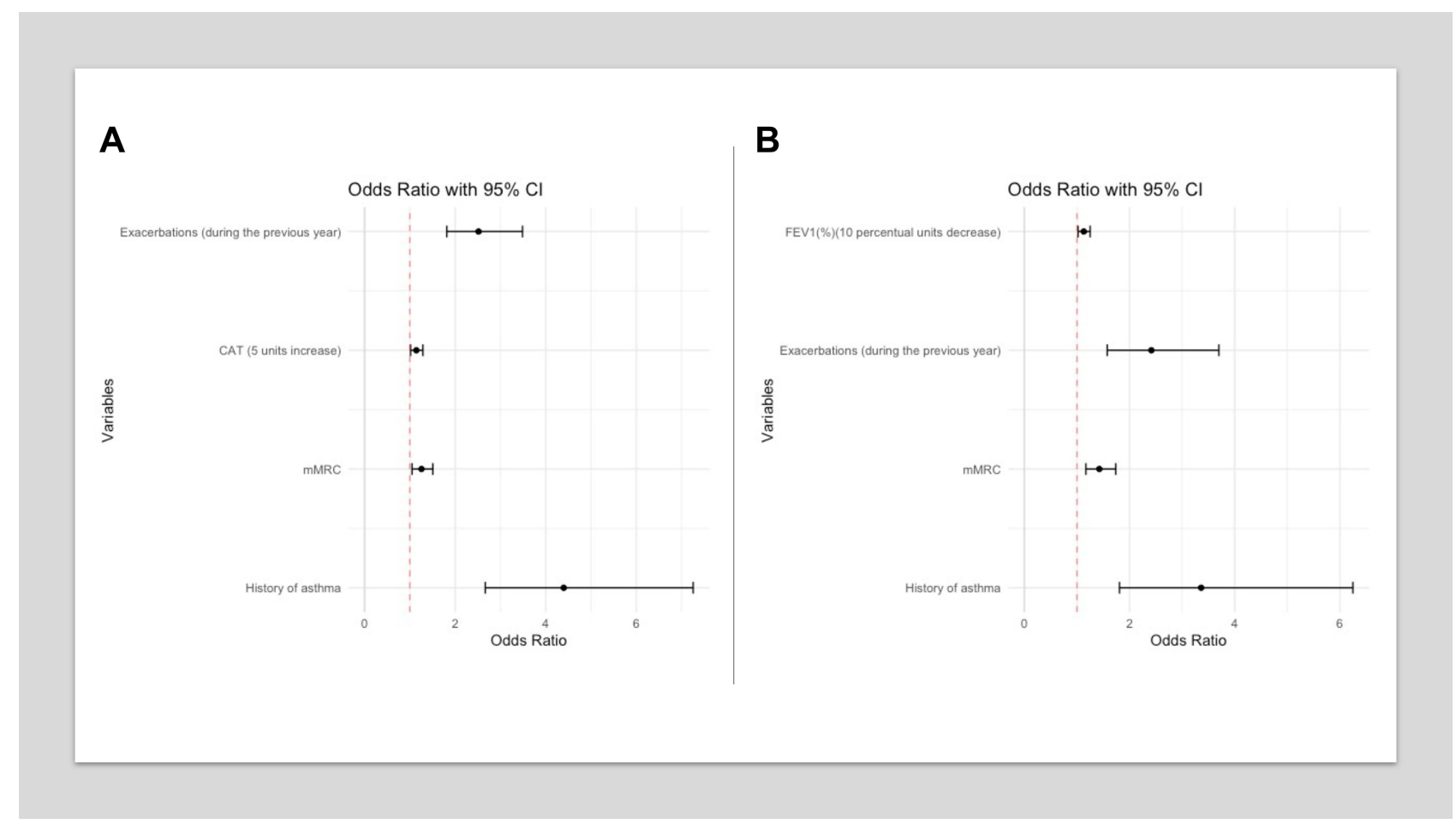

Figure 4 Forest plot of variables associated with the prescription of ICS for COPD in primary care. (A) Total population ( $\mathrm{n}=90 \mathrm{I})$, and $(\mathbf{B})$ population with spirometry confirmed COPD $(n=539)$.

Notes: (A) Dependent variable ICS yes/no and independent variables: age, sex, spirometry (yes/no), blood eosinophils (high/low), history of asthma (yes/no), mMRC, CAT (5 units increase), moderate/severe exacerbations previous year. Age, mMRC and CAT as continuous variables, Reference categories: gender (male), spirometry confirmed COPD (COPD), blood eosinophils (low number of eosinophils $<300$ cell $/ \mu \mathrm{L}$ or $<3.6 \%$ ), history of asthma (no), presence of moderate/severe exacerbations in the last year (no). OR for 2 grades increase in mMRC (OR=I.578 [95\% Cl: I.096-2.27I]). N=625 (276 patients without eosinophil values). (B) Dependent variable ICS yes/no and independent variables: age, sex, $\mathrm{FEV}_{1}(\%)$ ( 10 percentual units decrease), blood eosinophils (high/low), history of asthma (yes/no), mMRC, CAT, moderate/severe exacerbations previous year. Age, mMRC and CAT and $\mathrm{FEV}_{\mathrm{I}}(\%)$ as continuous variables. Reference categories: gender (male), eosinophils (low number of eosinophils), history of asthma (no), presence of moderate/severe exacerbations in the last year (no). N=407 because I 32 values had missing values for $\mathrm{FEV}$, $\%$ and/or eosinophils.

Abbreviations: CAT, COPD assessment test; $\mathrm{Cl}$, confidence interval; COPD, chronic obstructive pulmonary disease; ICS, inhaled corticosteroids; mMRC, modified Medical Research Council Dyspnea Scale; OR, odds ratio; FEV , forced expiratory volume in I second.

Co-existing asthma and exacerbation history were significantly associated with ICS prescription in primary care, revealing that the majority of ICS prescriptions were in line with guideline recommendations. Most asthma-COPD overlap patients were receiving ICS prescription according to current guidelines at the time when the study was conducted. The GOLD document suggests not to use the denomination ACO and refer the possible coexistence of asthma and COPD in a given patient. ${ }^{6}$ From our results, it is clear that this coexistence is one of the most relevant factors influencing the prescription of ICS in Primary Care. ${ }^{26}$ However, a great proportion of COPD patients were still not receiving a targeted prescription of ICS according to criteria proposed in current guidelines. In this sense, future interventions are needed to improve COPD patient care and promote adequate ICS use within the primary care setting, by prompting clinical guidance and education as acknowledged by previous effective interventions within this setting. ${ }^{27,28}$

We have observed a gradient in ICS use for increasing mMRC and CAT scores. To date, current recommendations do not support using ICS to improve shortness of breath or symptom control in COPD.${ }^{6-8}$ However, our findings emphasize poor adherence to these recommendations, and suggest that physicians could be inclined to prescribing ICS not only for prevention of exacerbations but also for symptom control. Our results concur with other recent studies that confirmed the association between symptoms and ICS prescription. ${ }^{29,30}$

Understanding the role of eosinophils in COPD has increased over the last years. ${ }^{31}$ Within our sample, blood eosinophils were not associated with ICS use, which in addition to the significant proportion of patient with missing eosinophil counts, could be suggesting a lack of awareness of the usefulness of eosinophil counts for the management of COPD within primary care. A recent meta-analysis concluded that blood eosinophil levels were a predictor of ICS 
response to prevent future exacerbations among COPD patients in post-hoc analyses of RCTs; however, this was not present in 4 of the 5 observational studies within the review, suggesting that in real-world clinical practice this correlation may not be so clear. ${ }^{32}$

Some limitations must be acknowledged. Some data used for this study relied on the data extracted from clinical records, which are subject to missing values and could reduce the conclusions' validity. However, most of the variables used for this study are frequently used during real clinical practice to manage COPD patients. To this end, variables of relevance, such as specific spirometry values and blood eosinophil counts, were unavailable for some patients within the cohort. While data quality could be the underlying reason for some missing values, which has been previously noted with primary care, these measures may also represent absent measures, adding to difficulties in establishing an adequate diagnosis. $^{33}$

\section{Conclusion}

Inhaled corticosteroids prescription continues to be generalized in Primary Care and higher than expected, considering treatment recommendations provided by clinical guidelines. Beyond history of asthma and exacerbations, ICS use was associated to mMRC and CAT scores, indicating that symptom control is likely to drive prescription within this setting. Better implementation of guidelines about the appropriate use of ICS in Primary Care is required.

\section{Abbreviations}

ACOS, asthma chronic obstructive pulmonary disease overlap syndrome; BMI, body mass index; BODEx, body mass index, obstruction, dyspnea and exacerbations index; CAT, chronic obstructive pulmonary disease assessment test; CI, confidence interval; COPD, chronic obstructive pulmonary disease; COTE, chronic obstructive pulmonary disease comorbidity test; ERS, European Respiratory Society; FEV ${ }_{1}$, forced expiratory volume in 1 second; FVC, forced vital capacity; GOLD, Global Initiative for Chronic Obstructive Lung Disease; ICS, inhaled corticosteroids; INE, Spanish National Institute of Statistics; LABA, long-acting beta2-agonist; LAMA, long-acting muscarinic antagonists; mMRC, modified Medical Research Council Dyspnea Scale; OR, odds ratio; SD, standard deviation.

\section{Data Sharing Statement}

To ensure independent interpretation of clinical study results, Boehringer Ingelheim grants all external authors access to all relevant material, including participant-level clinical study data, and relevant material as needed by them to fulfill their role and obligations as authors under the ICMJE criteria. Furthermore, clinical study documents (eg, study report, study protocol, statistical analysis plan) and participant clinical study data are available to be shared after publication of the primary manuscript in a peer-reviewed journal and if regulatory activities are complete and other criteria met per the BI Policy on Transparency and Publication of Clinical Study Data: https://trials.boehringer-ingelheim.com/. Prior to providing access, documents will be examined, and, if necessary, redacted, and the data will be de-identified, to protect the personal data of study participants and personnel, and to respect the boundaries of the informed consent of the study participants. Clinical Study Reports and Related Clinical Documents can also be requested via the link https://trials. boehringer-ingelheim.com/. All requests will be governed by a Document Sharing Agreement. Bona fide, qualified scientific and medical researchers may request access to de-identified, analysable participant clinical study data with corresponding documentation describing the structure and content of the datasets. Upon approval, and governed by a Data Sharing Agreement, data are shared in a secured data-access system for a limited period of 1 year, which may be extended upon request. Researchers should use the https://trials.boehringer-ingelheim.com/ link to request access to study data.

\section{Ethics Approval and Informed Consent}

The OPTI study was conducted in accordance with the protocol, the principles set forth in the Declaration of Helsinki, and the applicable Spanish regulations regarding the conduct of observational studies. All patients were required to sign the informed consent form to participate in this study. This study was approved by the Principality of Asturias' ethics committee. 


\section{Acknowledgments}

Authors would like to thank all the participating researchers of the OPTI study. Authors would also like to express their gratitude to Mireia Canals (Boehringer-Ingelheim) and Jordi Galera (TFS Health Science) for his scientific advice in the design and performance of the study.

\section{Author Contributions}

All authors made a significant contribution to the work reported, whether that is in the conception, study design, execution, acquisition of data, analysis and interpretation, or in all these areas; took part in drafting, revising or critically reviewing the article; gave final approval of the version to be published; have agreed on the journal to which the article has been submitted; and agree to be accountable for all aspects of the work.

\section{Funding}

The study was supported and funded by Boehringer - Ingelheim Spain. Julieta Politi of IQVIA provided medical writing and editorial support, which was contracted and funded by Boehringer- Ingelheim Spain.

\section{Disclosure}

Marc Miravitlles has received speaker fees from AstraZeneca, Boehringer Ingelheim, Chiesi, Cipla, GlaxoSmithKline, Menarini, Rovi, Bial, Sandoz, Zambon, CSL Behring, Grifols and Novartis, consulting fees from AstraZeneca, Atriva Therapeutics, Boehringer Ingelheim, Chiesi, GlaxoSmithKline, Bial, Gebro Pharma, CSL Behring, Laboratorios Esteve, Ferrer, Mereo Biopharma, Verona Pharma, Spin Therapeutics, ONO Pharma, pH Pharma, Palobiofarma SL, Takeda, Novartis, Sanofi and Grifols and research grants from Grifols. Miguel Roman-Rodriguez has received speaker fees from AstraZeneca, Boehringer Ingelheim, Chiesi, GlaxoSmithKline, Menarini, Bial, consulting fees from AstraZeneca, Boehringer Ingelheim, GlaxoSmithKline, and research grants from AstraZeneca in the last 3 years. Xavier Ribera is an employee of Boehringer Ingelheim Spain. John Ritz is an employee of Syneos Health - Boehringer Ingelheim Pharmaceuticals Inc. José Luis Izquierdo has received honoraria for consultancy, projects, and talks from AstraZeneca, Bayer, Boehringer Ingelheim, Chiesi, Glaxo, Grifols, Smith Kline, Menarini, Novartis, Orion, Pfizer, Sandoz, Teva, and Zambon. The authors report no other conflicts of interest in this work.

\section{References}

1. GBD 2015 Chronic Respiratory Disease Collaborators. Global, regional, and national deaths, prevalence, disability-adjusted life years, and years lived with disability for chronic obstructive pulmonary disease and asthma, 1990-2015: a systematic analysis for the Global Burden of Disease Study 2015. Lancet Respir Med. 2017;5(9):691-706. doi:10.1016/S2213-2600(17)30293-X

2. Soriano JB, Alfageme I, Miravitlles M, et al. Prevalence and determinants of COPD in Spain: EPISCAN II. Arch Bronconeumol. 2021;57(1):61-69. doi:10.1016/j.arbres.2020.07.024

3. Soler-Cataluna JJ, Martínez-García MA, Román Sánchez P, Salcedo E, Navarro M, Ochando R. Severe acute exacerbations and mortality in patients with chronic obstructive pulmonary disease. Thorax. 2005;60(11):925-931. doi:10.1136/thx.2005.040527

4. Halpin DM, Miravitlles M, Metzdorf N, Celli B. Impact and prevention of severe exacerbations of COPD: a review of the evidence. Int J Chron Obstruct Pulmon Dis. 2017;12:2891-2908. doi:10.2147/COPD.S139470

5. Alcázar Navarrete B, Ancochea Bermúdez J, García-Río F, et al. Patients with Chronic Obstructive Pulmonary Disease exacerbations: recommendations for diagnosis, treatment and care. Arch Bronconeumol. 2019;55(9):478-487. doi:10.1016/j.arbres.2019.02.020

6. Global Initiative for Chronic Obstructive Lung Disease [GOLD]. Global strategy for the diagnosis, management and prevention of Chronic Obstructive Lung Disease (2019 Report); 2019. Available from: https://goldcopd.org/wp-content/uploads/2018/11/GOLD-2019-v1.7-FINAL14Nov2018-WMS.pdf. Accessed July 20, 2021.

7. Miravitlles M, Vogelmeier C, Roche N, et al. A review of national guidelines for management of COPD in Europe. Eur Respir J. 2016;47 (2):625-637. doi:10.1183/13993003.01170-2015

8. Gazzotti MR, Roco CM, Pradella CO, et al. Frequency of osteoporosis and vertebral fractures in Chronic Obstructive Pulmonary Disease (COPD) patients. Arch Bronconeumol. 2019;55(5):252-257. doi:10.1016/j.arbres.2018.10.010

9. Miravitlles M, Auladell-Rispau A, Monteagudo M, et al. Systematic review on long-term adverse effects of inhaled corticosteroids in the treatment of COPD. Eur Respir Rev. 2021;30(160):210075. doi:10.1183/16000617.0075-2021

10. Miravitlles M, Calle M, Molina J, et al. Spanish COPD guidelines (GesEPOC) 2021: updated Pharmacological treatment of stable COPD. Arch Bronconeumol. 2022;58(1):69-81. doi:10.1016/j.arbr.2021.03.014

11. Miravitlles M, Barrecheguren M, Román-Rodríguez M. Frequency and characteristics of different clinical phenotypes of chronic obstructive pulmonary disease. Int J Tuberc Lung Dis. 2015;19(8):992-998. doi:10.5588/ijtld.15.0021 
12. Izquierdo JL, Morena D, González Y, et al. Clinical management of COPD in a real-world setting. A big data analysis. Arch Bronconeumol. 2021;57(2):94-100. doi:10.1016/j.arbres.2019.12.025

13. Chalmers JD, Laska IF, Franssen FME, et al. Withdrawal of inhaled corticosteroids in COPD: a European Respiratory Society guideline. Eur Respir J. 2020;55(6):2000351. doi:10.1183/13993003.00351-2020

14. Monteagudo M, Nuñez A, Solntseva I, et al. Treatment pathways before and after triple therapy in COPD: a population-based study in primary care in Spain. Arch Bronconeumol. 2021;57(3):205-213. doi:10.1016/j.arbres.2020.07.032

15. Instituto Nacional de Estadística (INE), Madrid, Spain. Available from: https:/www.ine.es/CDINEbase/consultar.do?mes=\&operacion=Cifras+de +Poblaci\%F3n\&id_oper=Ir. Accessed January 18, 2022.

16. Miravitlles M, Monteagudo M, Solntseva I, Alcázar B. Blood eosinophil counts and their variability and risk of exacerbations in COPD: a population-based study. Arch Bronconeumol. 2021;57(1):13-20. doi:10.1016/j.arbres.2019.12.015

17. Soler-Cataluña JJ, Martínez-García M, Sánchez Sánchez L, Perpiñá Tordera M, Román Sánchez P. Severe exacerbations and BODE index: two independent risk factors for death in male COPD patients. Respir Med. 2009;103(5):692-699. doi:10.1016/j.rmed.2008.12.005

18. Divo MJ, Casanova C, Marin JM, et al. COPD comorbidities network. Eur Respir J. 2015;46(3):640-650. doi:10.1183/09031936.00171614

19. Miravitlles M, Soler-Cataluña JJ, Calle M, et al. Spanish guidelines for management of Chronic Obstructive Pulmonary Disease (GesEPOC) 2017 pharmacological treatment of stable phase. Arch Bronconeumol. 2017;53(6):324-335. doi:10.1016/j.arbres.2017.03.018

20. Tsiligianni I, Kampouraki M, Ierodiakonou D, Poulonirakis I, Papadokostakis P. COPD patients' characteristics, usual care, and adherence to guidelines: the Greek UNLOCK study. Int J Chron Obstruct Pulmon Dis. 2019;14:547-556. doi:10.2147/COPD.S185362

21. Casas A, Montes de Oca M, Menezes A, et al. Respiratory medication used in COPD patients from seven Latin American countries: the LASSYC study. Int J Chron Obstruct Pulmon Dis. 2018;13:1545-1556. doi:10.2147/COPD.S154097

22. Price D, West D, Brusselle G, et al. Management of COPD in the UK primary-care setting: an analysis of real-life prescribing patterns. Int J Chron Obstruct Pulmon Dis. 2014;9:889-904. doi:10.2147/COPD.S62750

23. Román-Rodríguez M, van Boven JFM, Vargas F, et al. Factors associated with inhaled corticosteroids prescription in primary care patients with COPD: a cross-sectional study in the Balearic Islands (Spain). Eur J Gen Pract. 2016;22(4):232-239. doi:10.1080/13814788.2016.1212011

24. Nici L, Mammen MJ, Charbek E, et al. Pharmacologic management of Chronic Obstructive Pulmonary Disease. An official American thoracic society clinical practice guideline. Am J Respir Crit Care Med. 2020;201(9):e56-e69. doi:10.1164/rccm.202003-0625ST

25. Avdeev S, Aisanov Z, Arkhipov V, et al. Withdrawal of inhaled corticosteroids in COPD patients: rationale and algorithms. Int J Chron Obstruct Pulmon Dis. 2019;14:1267-1280. doi:10.2147/COPD.S207775

26. Miravitlles M, Andreu I, Romero Y, Sitjar S, Altés A, Anton E. Difficulties in differential diagnosis of COPD and asthma in primary care. Br J Gen Pract. 2012;62(595):e68-e75. doi:10.3399/bjgp12X625111

27. Cole JN, Mathur RA, Hull SA. Reducing the use of inhaled corticosteroids in mild-moderate COPD: an observational study in east London. NPJ Prim Care Respir Med. 2020;30(1):34. doi:10.1038/s41533-020-00191-y

28. Erro Iribarren M, Alonso Pérez T, Soriano JB, Ancochea Bermúdez J. Adjusting the Level of intervention in patients with Chronic Obstructive Pulmonary Disease according to the risk stratification proposed by the Spanish COPD guidelines (GesEPOC) version 2017. Arch Bronconeumol. 2020;56(3):183-185. doi:10.1016/j.arbres.2019.09.016

29. Monteagudo M, Barrecheguren M, Solntseva I, et al. Clinical characteristics and factors associated with triple therapy use in newly diagnosed patients with COPD. NPJ Prim Care Respir Med. 2021;31(1):16. doi:10.1038/s41533-021-00227-x

30. Bahremand T, Etminan M, Roshan-Moniri N, De Vera MA, Tavakoli H, Are SM. COPD prescription patterns aligned with guidelines? Evidence from a Canadian population-based study. Int J Chron Obstruct Pulmon Dis. 2021;16:751-759.

31. Asensio VJ, Tomás A, Iglesias A, et al. for CHACOS study group. Eosinophilic COPD patients display a distinctive serum miRNA profile from asthma and non-eosinophilic COPD. Arch Bronconeumol. 2020;56(4):234-241. doi:10.1016/j.arbres.2019.09.020

32. Harries TH, Rowland V, Corrigan CJ, et al. Blood eosinophil count, a marker of inhaled corticosteroid effectiveness in preventing COPD exacerbations in post-hoc RCT and observational studies: systematic review and meta-analysis. Respir Res. 2020;21(1):3.

33. Brouwer HJ, Bindels PJE, Weert HC. Data quality improvement in general practice. Fam Pract. 2006;23(5):529-536. doi:10.1093/fampra/cml040

International Journal of Chronic Obstructive Pulmonary Disease

Dovepress

\section{Publish your work in this journal}

The International Journal of COPD is an international, peer-reviewed journal of therapeutics and pharmacology focusing on concise rapid reporting of clinical studies and reviews in COPD. Special focus is given to the pathophysiological processes underlying the disease, intervention programs, patient focused education, and self management protocols. This journal is indexed on PubMed Central, MedLine and CAS. The manuscript management system is completely online and includes a very quick and fair peer-review system, which is all easy to use. Visit http://www. dovepress.com/testimonials.php to read real quotes from published authors.

Submit your manuscript here: https://www.dovepress.com/international-journal-of-chronic-obstructive-pulmonary-disease-journal 